\title{
Potential Role of Heme Oxygenase-1 in the Resolution of Experimentally Induced Colitis through Regulation of Macrophage Polarization
}

\author{
Shin-Young Gwak', Su-Jung Kim², Jeongmin Park', Seung Hyeon Kim4, Yeonsoo Joe², Ha-Na Lee ${ }^{5}$, Wonki Kim², \\ Ishrat Aklima Muna ${ }^{2}$, Hye-Kyung $\mathrm{Na}^{6}$, Hun Taeg Chung ${ }^{3}$, and Young-Joon Surh ${ }^{1,2,4}$ \\ ${ }^{1}$ Department of Molecular Medicine and Biopharmaceutical Sciences, Graduate School of Convergence Science and Technology, \\ ${ }^{2}$ Research Institute of Pharmaceutical Sciences, College of Pharmacy, Seoul National University, Seoul, ${ }^{3}$ Department of Biological \\ Sciences, University of Ulsan, Ulsan, ${ }^{4}$ Cancer Research Institute, Seoul National University, Seoul, ${ }^{5}$ Laboratory of Immunology, \\ Division of Biotechnology Review and Research-III, Office of Biotechnology Products, Center for Drug Evaluation and Research, Food \\ and Drug Administration, Silver Spring, MD, USA, and ${ }^{6}$ Department of Food Science and Biotechnology, College of Knowledge-Based \\ Services Engineering, Sungshin Women's University, Seoul, Korea
}

Article Info

Received February 5, 2021

Revised May 20, 2021

Accepted May 24, 2021

Published online November 5, 2021

Corresponding Author

Young-Joon Surh

ORCID https://orcid.org/0000-0001-8310-1795

E-mail surh@snu.ac.kr
Background/Aims: Heme oxygenase-1 (HO-1) plays a central role in cellular defense against inflammatory insults, and its induction in macrophages potentiates their efferocytic activity. In this study, we explored the potential role of macrophage $\mathrm{HO}-1$ in the resolution of experimentally induced colitis.

Methods: To induce colitis, male C57BL/6 mice were treated with $2 \%$ dextran sulfate sodium (DSS) in the drinking water for 7 days. To investigate efferocytosis, apoptotic colon epithelial CCD $841 \mathrm{CoN}$ cells were coincubated with bone marrow-derived macrophages (BMDMs).

Results: Administration of the HO-1 inhibitor zinc protoporphyrin IX (ZnPP) blunted the resolution of DSS-induced intestinal inflammation and expression of the proresolving M2 macrophage marker CD206. BMDMs treated with apoptotic colonic epithelial cells showed significantly elevated expression of $\mathrm{HO}-1$ and its regulator Nrf2. Under the same experimental conditions, the proportion of CD206-expressing macrophages was also enhanced. ZnPP treatment abrogated the upregulation of CD206 expression in BMDMs engulfing apoptotic colonic epithelial cells. This result was verified with BMDMs isolated from HO-1-knockout mice. BMDMs, when stimulated with lipopolysaccharide, exhibited increased expression of CD86, a marker of M1 macrophages. Coculture of lipopolysaccharide-stimulated BMDMs with apoptotic colonic epithelial cell debris dampened the expression of CD86 as well as the pro-inflammatory cytokines in an HO-1-dependent manner. Genetic ablation as well as pharmacologic inhibition of $\mathrm{HO}-1$ significantly reduced the proportion of efferocytic BMDMs expressing the scavenger receptor CD36.

Conclusions: $\mathrm{HO}-1$ plays a key role in the resolution of experimentally induced colitis by modulating the polarization of macrophages. (Gut Liver 2022;16:246-258)

Key Words: Acute colitis; Dextran sulfate sodium; Efferocytosis; Macrophage polarization; Resolution of inflammation

\section{INTRODUCTION}

Acute inflammation, a protective immune response provoked by infection- or injury-associated danger signals, should be terminated properly to restore the tissue homeo- stasis. ${ }^{1-3}$ Failure of resolution of acute inflammation causes the development of chronic diseases including arthritis, asthma, and atherosclerosis. ${ }^{4}$ Inflammatory bowel disease (IBD) is one of chronic inflammation-associated disorders influenced by environmental stressors, intestinal bacteria, 
an abnormal immune reaction, some genetic factors, etc. ${ }^{5-7}$ In a steady state, intestinal epithelial monolayer serves as a barrier between enteric microbiota and immune cell compartment in lamina propria. The chronic inflammatory conditions impair the entire gastrointestinal tract, rendering the underlying tissues more susceptible to pathogen infection continuously., ${ }^{5,8}$

Macrophages have important roles in preparative processes during the inflammation. Macrophages exert functions in inflamed tissues by converting themselves from a pro-inflammatory (M1) to an anti-inflammatory/proresolving (M2) phenotype. M2 macrophages secrete antiinflammatory cytokines such as interleukin 10 and growth factors. They also control extracellular matrix turnover and remodeling. ${ }^{3,7,10-15}$

Apoptotic cells are largely produced in infected and injured tissues. ${ }^{8,16}$ Phagocytic clearance of the apoptotic cell debris, termed "efferocytosis", is essential for preventing additive inflammatory reactions and autoimmunity. ${ }^{17-19}$ Notably, uptake of apoptotic cells promotes reprogramming of phagocytic macrophages toward the proresolving phenotype. ${ }^{20-23}$ Macrophage polarization during efferocytosis involves non-canonical mitochondrial response associated with fatty acid metabolism ${ }^{20}$ and autophagy. ${ }^{21}$

Heme oxygenase-1 (HO-1) is an inducible anti-oxidant and anti-inflammatory enzyme, and its expression is mainly regulated by nuclear factor-E2-related factor 2 (Nrf2), a master regulator of stress-responsive gene expression. ${ }^{24}$ HO- 1 has been reported to regulate inflammation by modulating production of anti-/pro-inflammatory cytokines in immune cells. ${ }^{25-29}$ Furthermore, HO-1 induction in macrophages promotes their phagocytic activity, ${ }^{30}$ and conversion of macrophages to an anti-inflammatory/proresolving phenotype..$^{25,27,31-33}$ In this study, we explored the role of HO-1 expressed in macrophages engulfing apoptotic colonic epithelial cells in the resolution of experimentally induced murine colitis.

\section{MATERIALS AND METHODS}

\section{Animals}

Male C57BL/6 mice (6-week-old) were purchased from Raon Bio (Seoul, Korea) and maintained according to the institutional animal care guidelines. Animal experimental procedures were approved by the Institutional Animal Care and Use Committee of Seoul National University (IACUC number: SNU-170725-7) and the University of Ulsan Animal Care and Use Committee (reference number: HTC-14-030).

\section{Reagents}

Dulbecco's Modified Eagle's Medium, Minimum Essential Media, and fetal bovine serum (FBS) were obtained from Gibco (Grand Island, NY, USA). Zinc protoporphyrin IX (ZnPP) and a primary antibody against $\mathrm{HO}-1$ were the products of Enzo Life Sciences (Farmingdale, NY, USA). Primary antibodies for detecting Nrf2 (ab137550) and CD11b (ab8878) were obtained from Abcam (Cambridge, MA, USA). Anti-rabbit and anti-mouse horseradish peroxidase-conjugated secondary antibodies were provided by Thermo Fisher Scientific (Eugene, OR, USA). Antibodies for TruStain FcX (anti-mouse CD16/32), CD45, CD11b, F4/80, Ly6C, Ly6G, CD86, CD36, and CD206 for flow cytometric analysis were purchased from Biolegend (San Diego, CA, USA). 4',6-Diamidino-2-phenylindole and phenylmethylsulfonyl fluoride were purchased from Thermo Fisher Scientific and Sigma-Aldrich (St. Louis, MO, USA), respectively.

\section{Induction of acute dextran sulfate sodium (DSS)- induced colitis}

Seven-week-old male C57BL/6 mice were treated with 2\% DSS (36-50 kDa; MP Biomedicals; Santa Ana, CA, USA) dissolved in drinking water for 7 days. Mice were euthanized by $\mathrm{CO}_{2}$ asphyxiation at various time intervals. When necessary, mice were daily injected intraperitoneally with vehicle or $\mathrm{ZnPP}$ (25 mg/kg) for 2 weeks after DSS administration.

\section{Histologic analysis}

Colons were removed from mice and rinsed with cold phosphate buffered saline (PBS) to remove luminal content. Tissue sections were fixed in $10 \%$ buffered formalin and embedded in paraffin. The sections were stained with hematoxylin and eosin. The histological scores were determined based on inflammatory cell infiltrates and integrity of the intestinal architecture according to the guideline described elsewhere. ${ }^{34}$

\section{Immunofluorescence}

Immunofluorescence was performed on murine colon tissue and isolated leukocytes. Colon-infiltrating myeloid cells were detected using rat monoclonal CD11b antibody (Abcam). Colonic epithelial cells were detected using rat Alexa Fluor $^{\circledR} 594$ anti-mouse CD326 (EpCAM) antibody (Biolegend). Immunofluorescence signals were visualized with secondary antibodies: Alexa Fluor ${ }^{\mathrm{TM}} 488$ goat anti-rat immunoglobulin G (IgG) (Thermo Fisher Scientific).

\section{Isolation of leukocytes from lamina propria}

To isolate lamina propria leukocytes, colons were 
flushed of their luminal content and opened longitudinally and cut into $1-\mathrm{cm}$ pieces. Epithelial cells were removed by 30-minute incubation with PBS containing 5\% FBS (Gibco), $2 \mathrm{mmol} / \mathrm{L}$ EDTA at $37^{\circ} \mathrm{C}$ under shaking at $275 \mathrm{rpm}$. Subsequent processes were performed as described previously. $^{35}$

\section{Preparation of bone marrow-derived macrophages (BMDMs)}

Bone marrow was harvested from femur and tibia of 8 - to 12 -week old mice by flushing marrow out with cold PBS. The cells from bone marrow were then filtered through a $70 \mu \mathrm{m}$ nylon cell strainer. Red blood cells were then removed by red blood cell lysis buffer (iNtRON Biotechnology; Seongnam, Korea). The remaining cells were resuspended in Dulbecco's Modified Eagle's Medium (Gibco) containing 10\% FBS and $20 \mathrm{ng} / \mathrm{mL}$ macrophage colony-stimulating factor (Biolegend), seeded in petri dishes and incubated at $37^{\circ} \mathrm{C}$ for 7 days in a humidified incubator containing 5\% $\mathrm{CO}_{2}$. Medium containing macrophage colony-stimulating factor $(10 \mathrm{ng} / \mathrm{mL})$ was changed once after 3 days of incubation.

\section{Measurement of CD206, CD86, and CD36 in BMDMs cocultured with apoptotic colon epithelial cells}

Apoptosis was induced in CCD 841 CoN colon epithelial cells by exposure to $180 \mathrm{~mJ}$ ultraviolet radiation 4 times and kept overnight at $37^{\circ} \mathrm{C}$ and $5 \% \mathrm{CO}_{2}$ for 24 hours. Cells were centrifuged at 1,200 rpm for 2 minutes, and pellets were washed 3 times with PBS. The apoptotic colon epithelial cells were added to the BMDMs (5:1 ratio) for 48 hours at $37^{\circ} \mathrm{C}$. To inhibit HO-1 activity, BMDMs were preincubated with $\mathrm{ZnPP}(10 \mu \mathrm{M})$ for 1 hour before the efferocytosis assay. After incubation, the mixtures were washed with PBS to remove free apoptotic cells and subjected to flow cytometry for the measurement of CD206 and CD36. After generating M1-like macrophages incubated with 100 $\mathrm{ng} / \mathrm{mL}$ of bacterial lipopolysaccharide (LPS) for 24 hours, the expression of CD86 was measured.

\section{Quantitative RT-PCR (qPCR) analysis}

Total RNA was isolated from cells with Trizol Reagent (Thermo Fisher Scientific) according to the manufacturer's protocol, and $1 \mu \mathrm{g}$ of RNA was reverse transcribed using the Moloney murine leukemia virus reverse transcriptase (Promega; Madison, WI, USA). Real-time quantitative PCR was performed on a 7300 Real-Time PCR instrument (Thermo Fisher Scientific) using the RealHelix Premier Quantitative PCR Kit (NanoHelix Co., Ltd.; Daejeon, Korea). The primers used for each RT-PCR reactions are as follows (forward and reverse, respectively): $\operatorname{Tnf}-\alpha$,
CCCTCACACTCAGATCATCTTCT and GCTACGACGTGGGCTACAG; Il12b, TGGTTTGCCATCGTTTTGCTG and ACAGGTGAGGTTCACTGTTTG. Gapdh gene was amplified as internal control. Data was analyzed using the comparative $\mathrm{C} \tau$ method.

\section{Flow cytometry}

BMDMs and single cells isolated from colon tissue were incubated with TruStain FcX (anti-mouse CD16/32) (Biolegend) in staining buffer containing 1\% FBS and $0.1 \%$ $\mathrm{NaN}_{3}$ in PBS to block nonspecific binding to Fc receptor. Specific antibodies for membrane markers (anti-mouse CD45 APC, CD11b Alexa Fluor ${ }^{\mathrm{TM}}$ 700, F4/80 PerCP/Cy5, Ly6C PE/Cy7, Ly6G APC/Cy7 and CD206 FITC) (Biolegend) were added to samples in the presence of CD16/32 antibody and incubated for 1 hour at $4{ }^{\circ} \mathrm{C}$. 4,6-Diamidino2-phenylindole was added to the samples about 10 minutes before flow cytometry analysis to gate out dead cells. Gating strategies to identify intestinal macrophages are illustrated in Supplementary Fig. 1. Sequential gating was used to identify specific cell populations. The dead cells were excluded using the viability dye, 4,6-diamidino-2-phenylindole. The leukocytes were identified and gated by the expression of CD45. The myeloid cells were then further identified and gated by the expression of CD11b. The F4/80 positive macrophages were gated into Ly6G negative and Ly6C negative population to exclude granulocytes and undifferentiated monocytes respectively.

To measure the efferocytic activity of macrophages isolated from colon tissue, EpCAM was used as a marker of colonic epithelial cell debris. Cells were fixed with $4 \%$ formaldehyde in PBS for 30 minutes at room temperature and stained with the macrophage marker, F4/80. After permeabilization with fixation/permeabilization buffer set (Thermo Fisher Scientific; Waltham, MA, USA), the cells were stained with anti-mouse EpCAM FITC (Biolegend).

To evaluate the expression of HO-1 in colonic macrophages, cells were fixed and permeabilized as described above. Cells were then incubated with $\mathrm{HO}-1$ primary antibody and goat anti-mouse IgG Fc Cross-Adsorbed secondary antibody (Invitrogen; Rockford, IL, USA). Cells were analyzed by FACS Calibur, LSR Fortessa X-20 and FACS Aria III (BD; Franklin Lakes, NJ, USA) machines. FlowJo software (Ashland, OR, USA) was used to analyze the data.

\section{Western blot analysis}

Mouse colon tissues were homogenized in ice-cold $1 \mathrm{X}$ lysis buffer (20 mM Tris- $\mathrm{HCl}$ [pH 7.5], $150 \mathrm{mM} \mathrm{NaCl}$, $1 \mathrm{mM} \mathrm{Na} \mathrm{EDDTA}_{2} 1 \mathrm{mM}$ EGTA, 1\% Triton, $2.5 \mathrm{mM}$ sodium pyrophosphate, $1 \mathrm{mM} \beta$-glycerophosphate, $1 \mathrm{mM} \mathrm{Na}_{3} \mathrm{VO}_{4}$, $1 \mathrm{mg} / \mathrm{mL}$ leupeptin) supplemented with a protease inhibi- 
tor and $0.1 \mathrm{mM}$ phenylmethylsulfonyl fluoride. After periodical vortex mixing for 2 hours at $4^{\circ} \mathrm{C}$, the lysates were centrifuged for 15 minutes at $12,000 \mathrm{~g}$.

BMDMs were collected and treated with the same cell lysis buffer containing $0.1 \mathrm{mM}$ phenylmethylsulfonyl fluoride and protease inhibitor for overnight at $4^{\circ} \mathrm{C}$, followed by centrifugation for 15 minutes at $12,000 \mathrm{~g}$. The cell lysates were subjected to SDS-polyacrylamide gel electrophoresis according to the standard protocol.

\section{Statistical analysis}

All data are presented as mean \pm standard error of the mean. Statistical significance was performed using the Student t-test or one-way analysis of variance with Tukey's multiple-comparisons post hoc test. All experiments were repeated at least 3 times unless specified. Differences were considered statistically significant at $\mathrm{p}<0.05$.

\section{RESULTS}

\section{Mice recover from DSS-induced acute colitis}

Murine colitis was induced according to the schedule as described in Materials and Methods. DSS-induced intestinal inflammation damages epithelial monolayer lining of colonic wall. ${ }^{36,37}$ The histological assessment of hematoxy- lin and eosin-stained tissue sections revealed massive disruption of the epithelial crypt shape which was gradually restored from day 10 (Fig. 1A). DSS-induced acute colitis was almost completely resolved by day 21 . DSS-treated mice lost body weight from day 5 to 9 , which was recovered gradually thereafter (Fig. 1B). Body weight loss was also associated with shortening of the colon (Supplementary Fig. 2).

\section{DSS-induced acute colitis and its resolution are accompanied by differential profiles of leukocyte recruitment}

Immune cells in lamina propria were isolated from colon tissue of mice after DSS administration. The proportion of leukocyte subsets in live cells was analyzed by flow cytometry. The leukocyte gating strategy is schematically presented in Supplementary Fig. 1. The increased proportion of $\mathrm{CD} 45^{+}$total leukocytes was sustained from days 10 to 17 . The percentage of $\mathrm{CD} 45^{+} \mathrm{CD} 11 \mathrm{~b}^{+}$myeloid lineage cells gradually increased from day 7 to 14 . The proportion of $\mathrm{CD}_{4} 5^{+} \mathrm{CD} 11 \mathrm{~b}^{+} \mathrm{Ly} 6 \mathrm{C}^{+}$infiltrated monocytes and $\mathrm{CD} 45^{+} \mathrm{CD} 11 \mathrm{~b}^{+} \mathrm{Ly} 6 \mathrm{G}^{+}$granulocytes escalated from day 7 and peaked on day 14, while that of macrophages peaked on day 14 and then decreased (Fig. 1C).

A
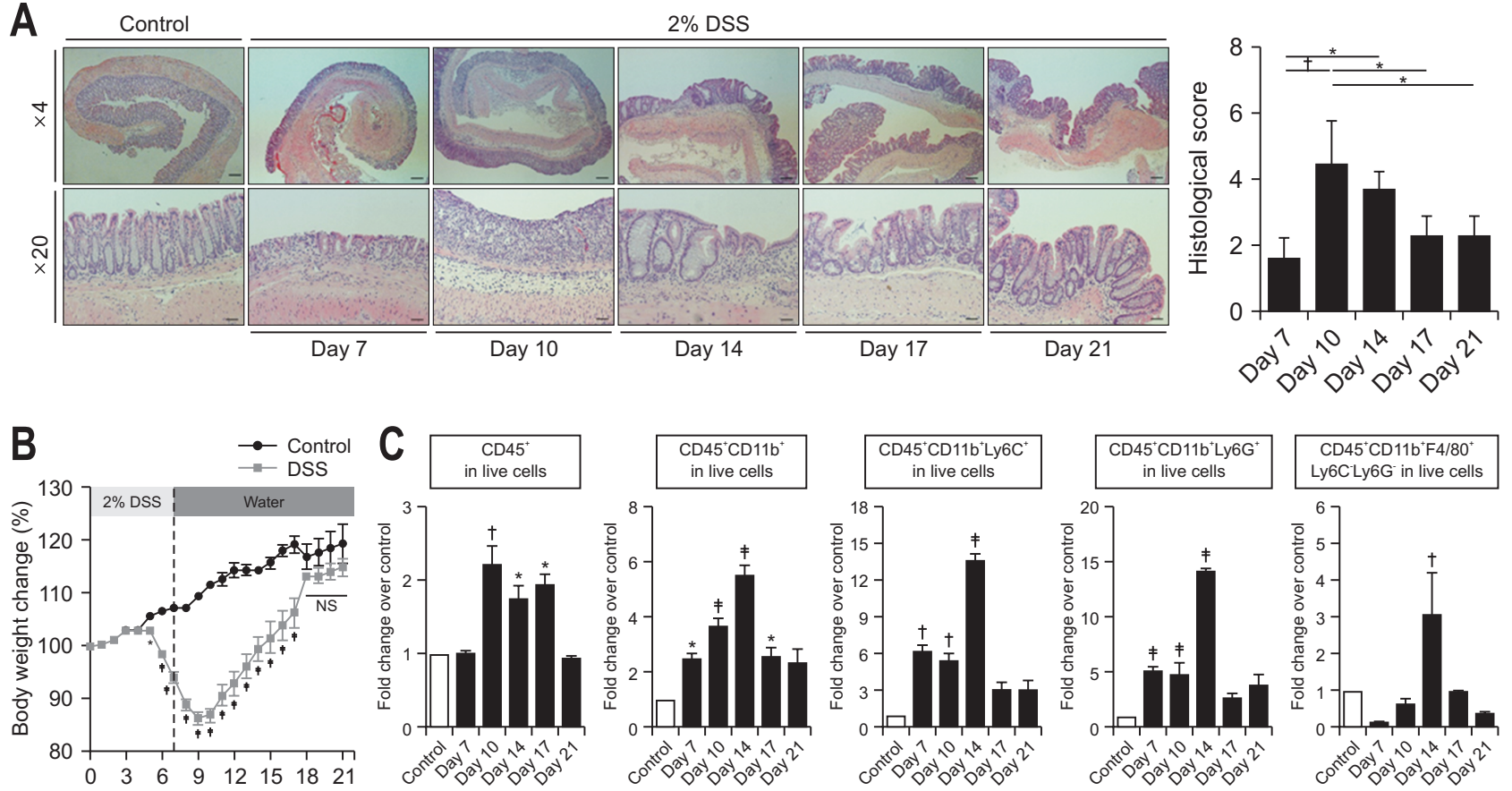

Fig. 1. The resolution of dextran sulfate sodium (DSS)-induced colitis. (A) Representative hematoxylin and eosin staining of colonic tissue sections. Scale bars correspond to $200 \mu \mathrm{m}$ (upper) and $40 \mu \mathrm{m}$ (lower). (B) Body weight changes. (C) Relative ratio of infiltrated leukocytes in colonic tissue from the indicated group of mice. Data are expressed as the mean \pm SEM $(n=3)$.

NS, no significance; SEM, standard error of the mean. ${ }^{*} p<0.05,{ }^{\dagger} p<0.01,{ }^{\ddagger} p<0.001$. 


\section{HO-1 is involved in resolution of DSS-induced colitis}

HO- 1 catalyzes the reaction that degrades the heme group present in various proteins including hemoglobin. HO-1 has been shown to ameliorate the damaging immune response in several models of intestinal inflammation. ${ }^{38}$ DSS administration resulted in transient upregulation of HO-1 expression in the colonic mucosa of mice (Fig. 2A) as part of host adaptive survival response to colitis. To examine the role of HO-1 during the resolution of colitis, we utilized the HO-1 inhibitor, ZnPP. After exposure to DSS for 7 days, ZnPP was intraperitoneally injected daily for 2 weeks. As shown in Fig. 2B, the survival rate was dramatically decreased in ZnPP-treated mice while none of mice administered DSS alone died. The mice in the ZnPPtreated group restored their body weight, which had been lost by the DSS administration, to a much lesser extent than did those in the vehicle-treated group (Fig. 2C). Pharmacologic inhibition of HO-1 also delayed the recovery from inflammatory injuries in colonic mucosa as assessed by histologic examination (Fig. 2D). The elevated infiltration of leukocytes caused by DSS gradually returned to the control level in vehicle-treated mice, but ZnPP-treated mice showed a higher level of infiltrated total leukocytes and cells of myeloid lineages. Among the myeloid lineage cells, macrophages were mainly accumulated in the colon tissue of ZnPP-treated mice during the resolution phase (Supplementary Fig. 3).

Macrophages orchestrate restoration of the tissue structure and homeostasis by changing their phenotypes from a pro-inflammatory (M1) to an anti-inflammatory/ proresolving (M2) state. Macrophage reprogramming for tissue repair is a dynamic and plastic process and can be
A
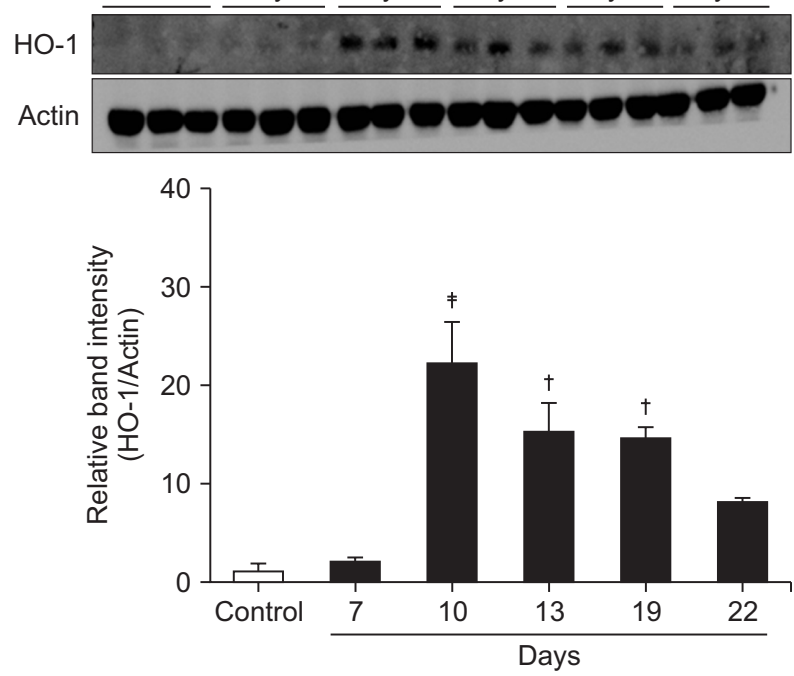

B

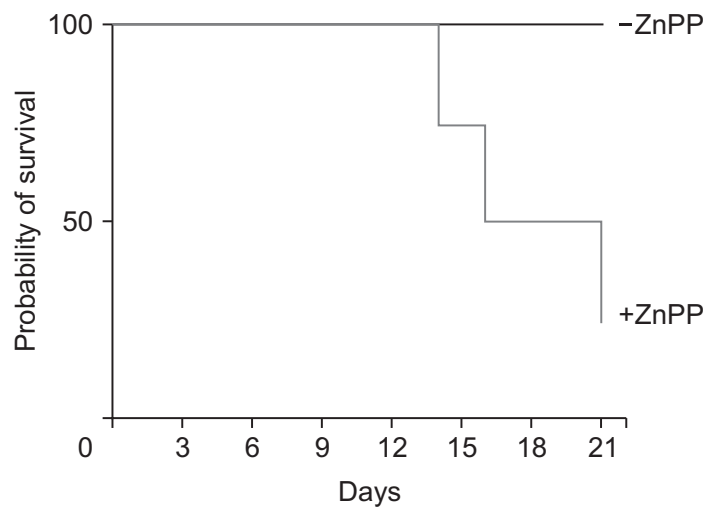

C
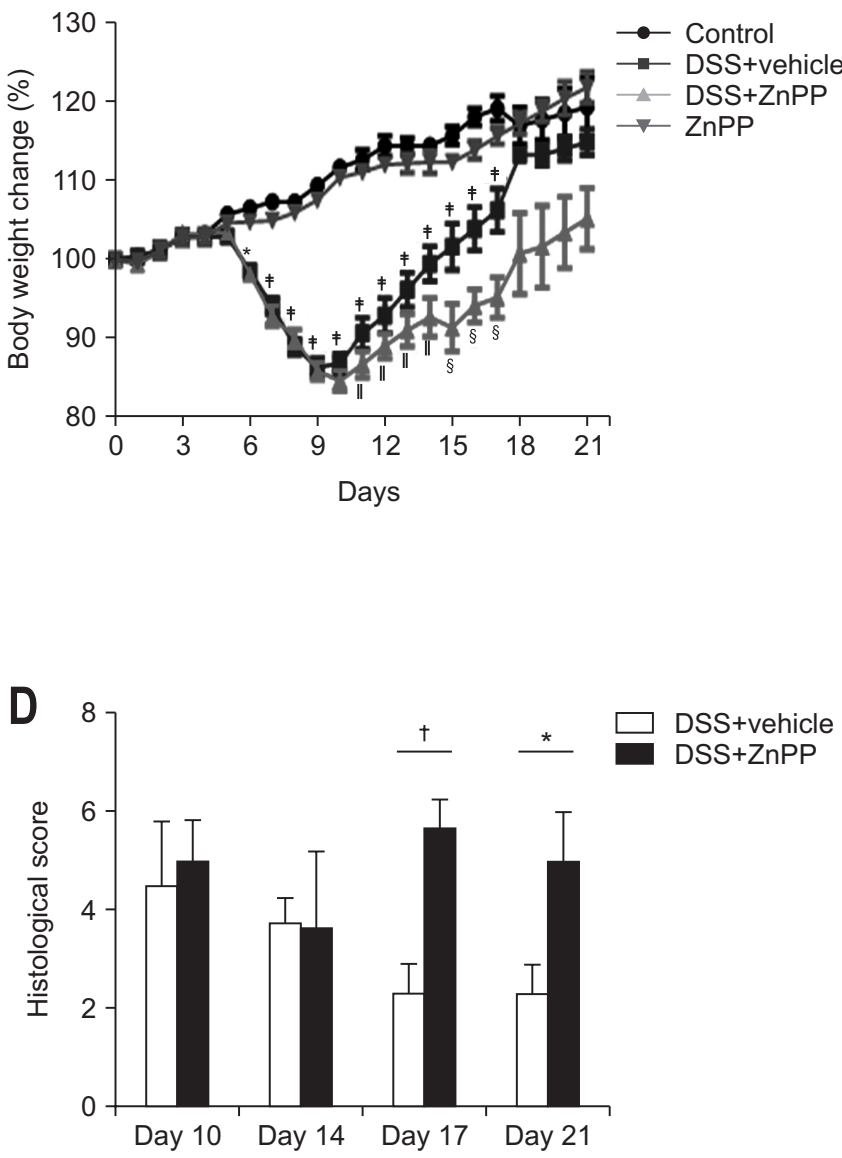

Fig. 2. The inhibition of heme oxygenase-1 (HO-1) activity delays the resolution of dextran sulfate sodium (DSS)-induced colitis. Mice were provided drinking water containing $2 \%$ DSS for 7 days, followed by regular water for another 14 days. Mice were daily injected with vehicle or 25 mg/kg bodyweight of zinc protoporphyrin IX (ZnPP) after termination of DSS administration. (A) Western blot analysis was performed to detect the expression of $\mathrm{HO}-1$ in whole colon tissue. (B) Survival rates. (C) Body weight changes. (D) Histologic scores. Data are expressed as the mean $\pm \mathrm{SEM}$ ( $\mathrm{n}=3$ ) SEM, standard error of the mean. Significantly different from the control ( $\left.{ }^{*} p<0.05,{ }^{+} p<0.01,{ }^{\ddagger} p<0.001\right)$; Significantly different from the DSS + vehicle group $\left({ }^{\S} p<0.05, " \| p<0.001\right)$. 
promoted by phagocytosing dead cells. ${ }^{3,16,20-22,39}$ In DSSinduced colitis, the expression of an M2 marker (CD206) in colonic macrophages gradually increased until day 14 and then decreased (Fig. 3A). As shown in Fig. 3B, expression of macrophage CD206 was significantly repressed in the colon of ZnPP-treated mice.

\section{The expression of HO-1 is upregulated in macrophages engulfing apoptotic colonic epithelial cells}

Our previous studies have demonstrated that HO-1 plays a vital role in efferocytic ${ }^{40}$ and phagocytic ${ }^{41}$ activities of macrophages. It has been reported that colonic mucosal $\mathrm{CX}_{3} \mathrm{CR}_{1}{ }^{+}$macrophages are the main producer of HO-1 in intestinal inflammation. ${ }^{29}$ In line with this notion, the proportion of colonic macrophages expressing HO- 1 was also escalated concomitantly with beginning of resolution (Fig. 3C).

\section{M2 macrophage polarization is controlled by HO-1 during the engulfment of apoptotic colonic epithelial cells}

In the resolution phase, phagocytic removal of apoptotic cells through efferocytosis prevents secondary necrosis of apoptotic cells which triggers an additive inflammatory reaction. ${ }^{1,3,7,42}$ Immunofluorescence staining performed on fixed leukocytes isolated from murine colitis showed that myeloid cells from both normal and inflammatory colonic tissue similarly participated in engulfing epithelial cells (Fig. 4A 2nd and 4th panels). However, the number of myeloid cells was found to be higher in inflamed colonic tissue compared with that of normal colonic tissue on day 10 (Fig. 1C 2nd panel, and Fig. 4A 1st and 3rd panels). There was a concomitant increase in the proportion of macrophages phagocytosing epithelial cells at the beginning of the resolution phase (Fig. 4B).

To confirm the direct association between efferocytosis and HO- 1 induction in macrophages, BMDMs isolated from mouse femurs were treated with apoptotic colonic epithelial cells generated by ultraviolet irradiation. Ultraviolet-induced cell death was confirmed by the poly (ADPribose) polymerase cleavage (Fig. 4C). As a result, the expression of HO-1 and its regulator, Nrf2 was significantly upregulated (Fig. 4D). Coculture of BMDMs with apoptotic colonic epithelial cell debris enhanced expression of the M2 macrophage marker, CD206 (Fig. 5). However, BM-
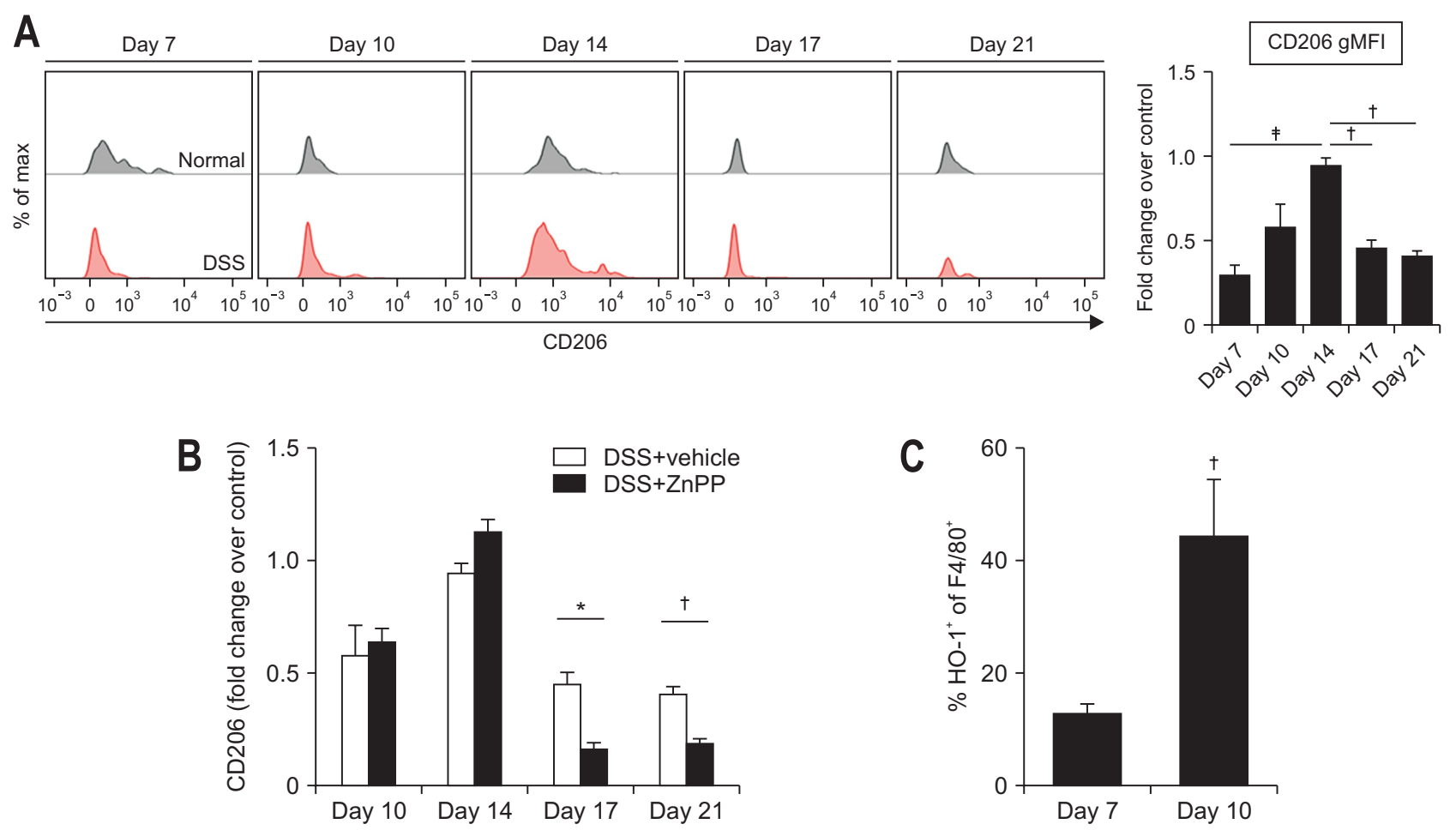

Fig. 3. The enhanced expression of CD206 and heme oxygenase-1 (HO-1) in macrophages of dextran sulfate sodium (DSS)-treated mouse colons. (A) Flow cytometric analysis of CD206 expression in macrophages from the colonic tissue of mice with or without DSS administration. (B) Effects of zinc protoporphyrin IX (ZnPP) on the expression of CD206 in colonic tissue macrophages of DSS-treated mice. Mice were injected intraperitoneally daily with vehicle or $\mathrm{ZnPP}$ ( $25 \mathrm{mg} / \mathrm{kg}$ ) for 2 weeks after DSS administration. (C) Flow cytometric analysis of HO- 1 expression in F4/80 ${ }^{+}$macrophages. Data are expressed as the mean $\pm S E M(n=3)$. gMFI, geometric mean fluorescence intensity; SEM, standard error of the mean. ${ }^{*} p<0.05,{ }^{\dagger} p<0.01,{ }^{\ddagger} p<0.001$. 
A

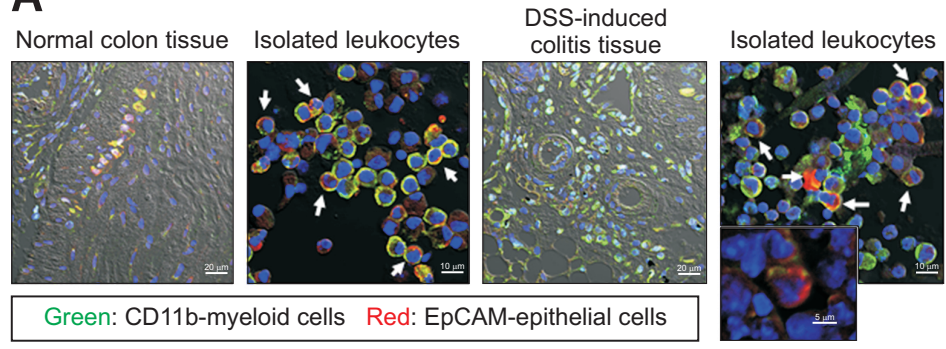

B

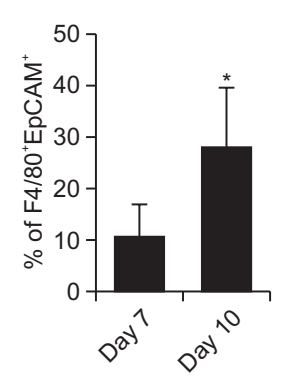

C

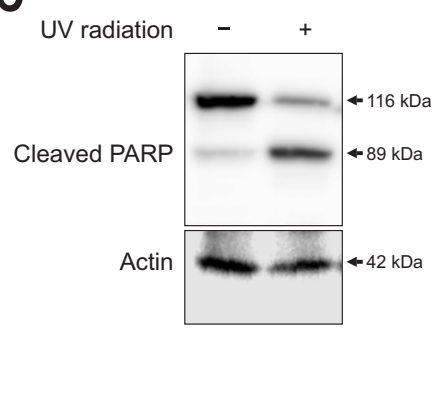

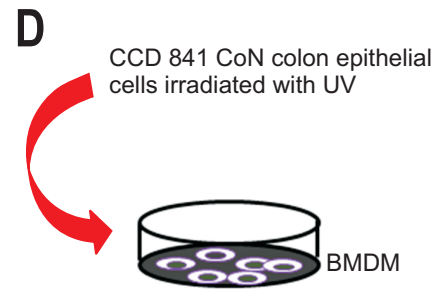
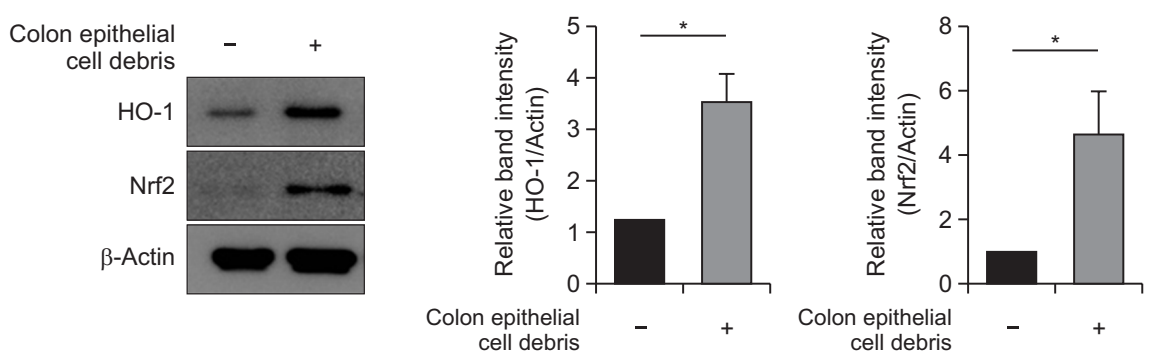

Fig. 4. Engulfment of apoptotic epithelial cells upregulates heme oxygenase-1 (HO-1) expression in macrophages. (A) Immunofluorescence images of fixed colonic tissue sections (1st and 3rd panels) and fixed single myeloid cells (2nd and 4th panels). Myeloid cells isolated from colitis tissues of dextran sulfate sodium (DSS)-treated mice or normal colon tissues were cytospun onto glass slides and subjected to immunofluorescence analysis for detection of colon epithelial cell debris (EpCAM ${ }^{+}$, red) engulfed by myeloid cells (CD11 b ${ }^{+}$, green). Arrows indicate myeloid cells engulfing colonic epithelial cells. (B) Flow cytometric analysis of macrophages engulfing colonic epithelial cells. (C) Verification of apoptotic death in colonic epithelial cells by Western blot analysis of cleaved PARP. (D) Bone marrow-derived macrophages (BMDMs) were cocultured with colonic epithelial cell debris killed by ultraviolet (UV) radiation and then subjected to Western blot analysis to measure the expression of $\mathrm{HO}$ - 1 and its transcription factor nuclear factor-E2-related factor 2 (Nrf2). Data are expressed as the mean $\pm S E M(n=3)$.

PARP, poly (ADP-ribose) polymerase; SEM, standard error of the mean. ${ }^{*} \mathrm{p}<0.05$.

DMs engulfing apoptotic colonic epithelial cells attained phenotypic characteristics of M2 macrophages to a lesser extent in the presence of ZnPP (Fig. 5A). The mechanistic dependency of macrophages engulfing dead epithelial cells on $\mathrm{HO}-1$ in their polarization was verified by using BMDMs isolated from HO-1 knockout (KO) mice (Fig. 5B). In contrast, the expression of an M1 macrophage marker CD86 was reduced in LPS-stimulated BMDMs cocultured with apoptotic colonic epithelial cells, which was abrogated in HO-1 deficient mice (Fig. 6A).

M1 macrophages secrete tumor necrosis factor $\alpha$ and interleukin-12, two major macrophage-derived mediators of inflammatory responses in mammals. ${ }^{25}$ After coculture of LPS-stimulated wild type murine BMDMs with apoptotic CCD $841 \mathrm{CoN}$ cell debris, there was a marked decrease in the expression of messenger RNA transcripts of pro-inflammatory M1 markers, Tnf- $\alpha$ (Fig. 6B) and Il12b (Fig. 6C), but this was attenuated in the BMDMs from HO-1 knockout mice.

$\mathrm{CD} 36$ is a prototypic scavenger receptor that is involved in the recognition of apoptotic cells by macrophages. CD36 present on the surface of macrophages mediates efferocytosis following tissue injury and thereby prevents excessive inflammation that could compromise tissue repair. ${ }^{43}$ The role of CD36 in the pathophysiology of IBD has been suggested. ${ }^{44} \mathrm{CD} 36$ deficiency aggravated DSS-induced colitis. Interestingly, we found that CD36 expression is upregulated in wild type BMDMs stimulated by the apoptotic epithelial cell debris, and this was blunted in BMDMs treated with the HO-1 inhibitor, $\mathrm{ZnPP}$ and also in those from HO-1 knockout mice (Fig. 7).

\section{DISCUSSION}

In order to investigate the pathophysiological features and therapeutic strategies for the treatment of IBD, several animal models have been developed. ${ }^{45}$ DSS is one of the most widely used chemical colitogens. This high molecular weight sulfated polysaccharide, when administered to mice as a dissolved form in drinking water, causes disruption of intestinal epithelial monolayer. The resulting pathological features mimic the superficial inflammation seen in ulcerative colitis (UC). ${ }^{35,36,40}$

The recruitment as well as origin of intestinal macrophages is highly dynamic. ${ }^{46}$ In other organs, tissue resident macrophages are not derived from monocytes, but rather from embryonic progenitors arising from the yolk sac and/ 
A

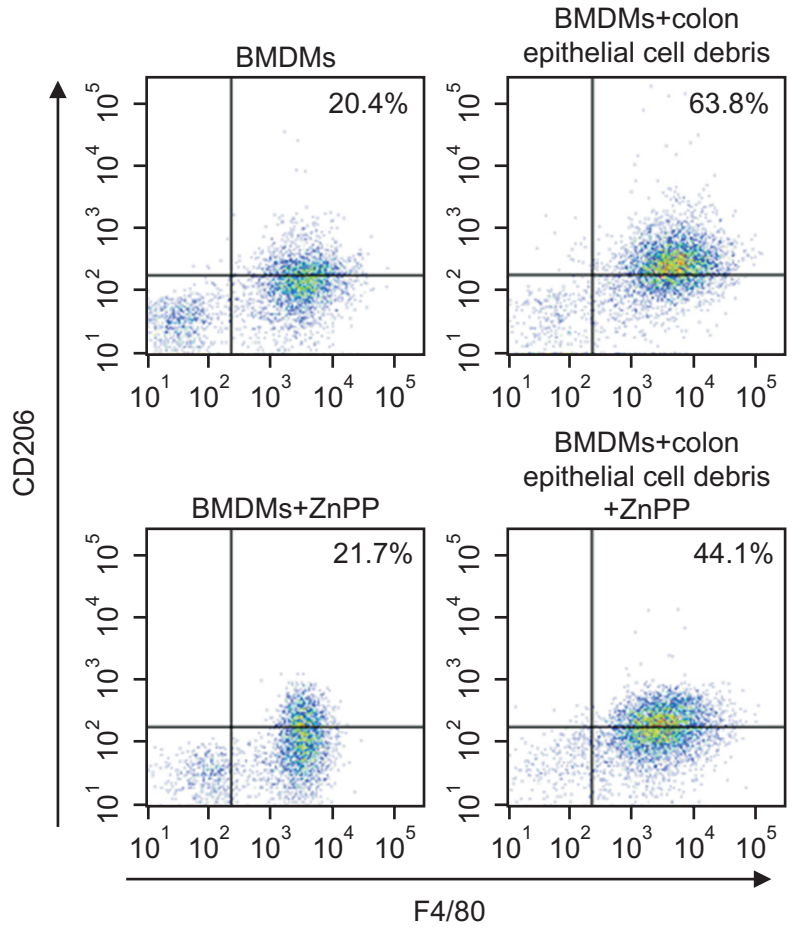

B
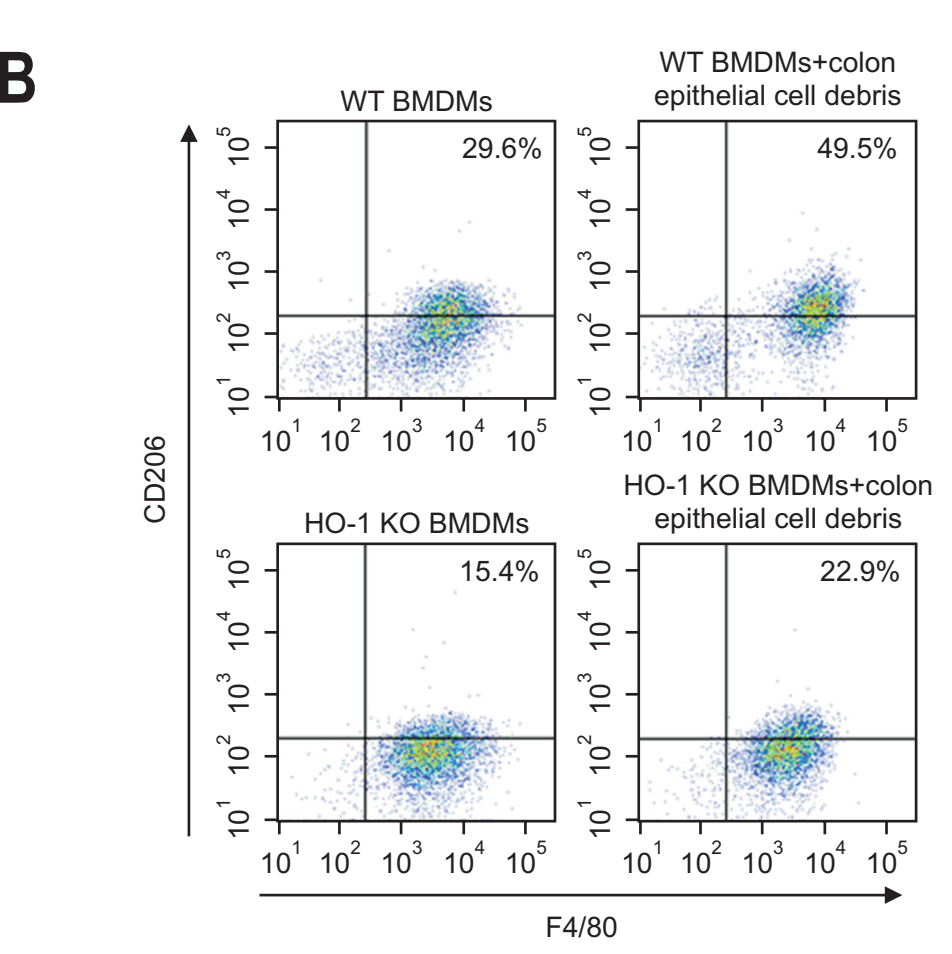

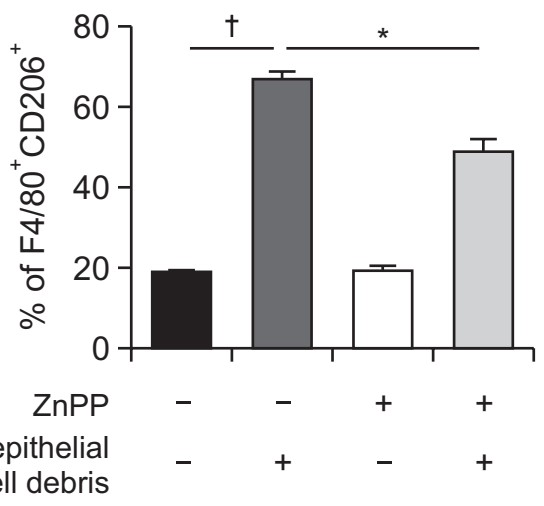

Colon epithelial cell debris

Fig. 5. Role of heme oxygenase-1 (HO-1) in M2-polarized bone marrow-derived macrophages (BMDMs) engulfing apoptotic colonic epithelial cells. CD206 expression in BMDMs mixed with apoptotic epithelial cells in two ways to examine the role of HO- 1 in vitro. (A) Murine BMDMs were treated with a pharmacological HO-1 inhibitor, zinc protoporphyrin IX (ZnPP) (10 mM), 1 hour prior to the addition of apoptotic epithelial cells. After incubation for 48 hours, the proportion of BMDMs expressing CD206 was measured by flow cytometry. (B) BMDMs from HO-1 knockout (KO) mice were treated with apoptotic epithelial cells and subjected to flow cytometry. Data are expressed as the mean $\pm \mathrm{SEM}(\mathrm{n}=3)$.

WT, wild type; SEM, standard error of the mean. ${ }^{*} p<0.01,{ }^{+} p<0.001$.

or fetal liver. They have been known to have a key function in homeostasis and inflammation. ${ }^{46-48}$ However, intestinal macrophages are largely replaced by Ly6C $C^{\text {high }}$ monocytes- derived macrophages in adulthood, ${ }^{49}$ implying that embryonic macrophages and BMDMs are present together in the gut wall. ${ }^{46}$ Especially in an inflammatory environ- 
A
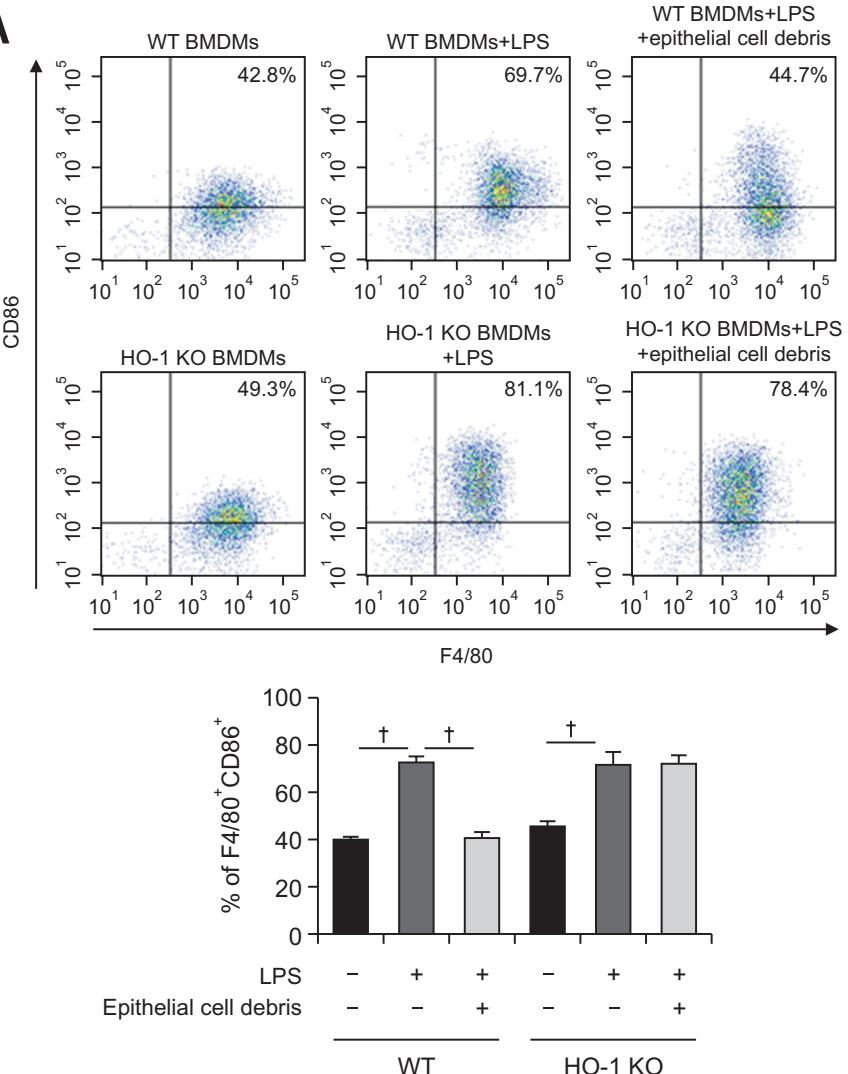

B

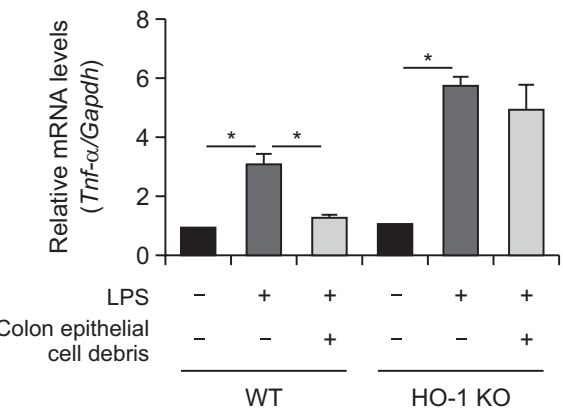

C

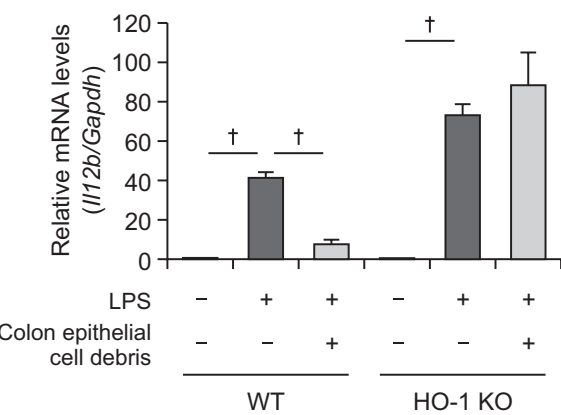

Fig. 6. Role of heme oxygenase-1 (HO-1) in M1-polarized bone marrow-derived macrophages (BMDMs) phagocytosing colonic epithelial cell debris. (A) BMDMs from wild type (WT) or H0-1 knockout (KO) mice were cocultured with or without CCD 841 CoN colon epithelial cell debris. After 48 hours of incubation, the proportion of macrophages expressing CD86 was assessed by flow cytometry. (B, C) BMDMs derived from WT or HO-1 $\mathrm{KO}$ mice were treated with lipopolysaccharide (LPS) $(100 \mathrm{ng} / \mathrm{mL})$ for 24 hours. The messenger RNA expression levels of Tnf- $\alpha$ (B) and $/(12 b(C)$ in the same macrophages cocultured with or without apoptotic epithelial cell debris for 48 hours were measured by quantitative reverse transcription polymerase chain reaction. ${ }^{*} p<0.01,{ }^{\dagger} p<0.001$.

ment including DSS-induced colitis, colonic tissues show intense accumulation of monocytes and immature macrophages. ${ }^{46,50,51}$ Consistent with these observations, leukocyte dynamics in the present study also show the accumulation of infiltrated cells of myeloid lineage, including monocytes, granulocytes and macrophages during resolution of DSSinduced colitis.

Macrophage infiltration and recruitment are common features of the early phase of inflammatory insult responsible for tissue injury, but these also lead to resolution of inflammation and tissue repair. ${ }^{52}$ Depending on the inflammatory milieu, macrophages undergo polarization to one of two phenotypes, classical or pro-inflammatory M1-like and alternatively activated or anti-inflammatory/ proresolving M2-like macrophages. Elevated M1 and concomitantly decreased M2 macrophages are commonly observed in the colonic tissues of UC patients. ${ }^{53}$ Likewise, macrophages have opposing roles in DSS-induced murine colitis that mimics UC. Thus, M1 macrophages contribute to the pathogenesis of colitis whilst M2 macrophages elicit protective functions by promoting tissue repair and by driving epithelial cell regeneration and proliferation. ${ }^{54}$ When macrophages fail to switch from M1 to M2 polarization, severe colitis arises in mice. ${ }^{55}$

Our present study demonstrated the accumulation of phagocytic M2 macrophages $\left(\mathrm{F} 4 / 80^{+} \mathrm{CD} 206^{+}\right)$in the mouse colon during the resolution of DSS-induced intestinal inflammation and also polarization of isolated BMDMs engulfing colonic epithelial cell debris toward the M2 phenotype, which appeared to be associated with the macrophage HO-1 activity. BMDMs, when stimulated with LPS, exhibited the increased expression of CD86, one of the typical M1 markers. Coculture of LPS-stimulated BMDMs with apoptotic colonic epithelial cell debris dampened the expression of CD86 as well as the genes encoding proinflammatory cytokines (tumor necrosis factor $\alpha$ and IL12p40) in an HO-1-dependent way.

HO-1 induction occurs in cells and tissues where hemoglobin is degraded by macrophages. ${ }^{38}$ Bacterial endotoxins or invasive pathogens can also induce $\mathrm{HO}-1 \mathrm{ex}$ - 
A

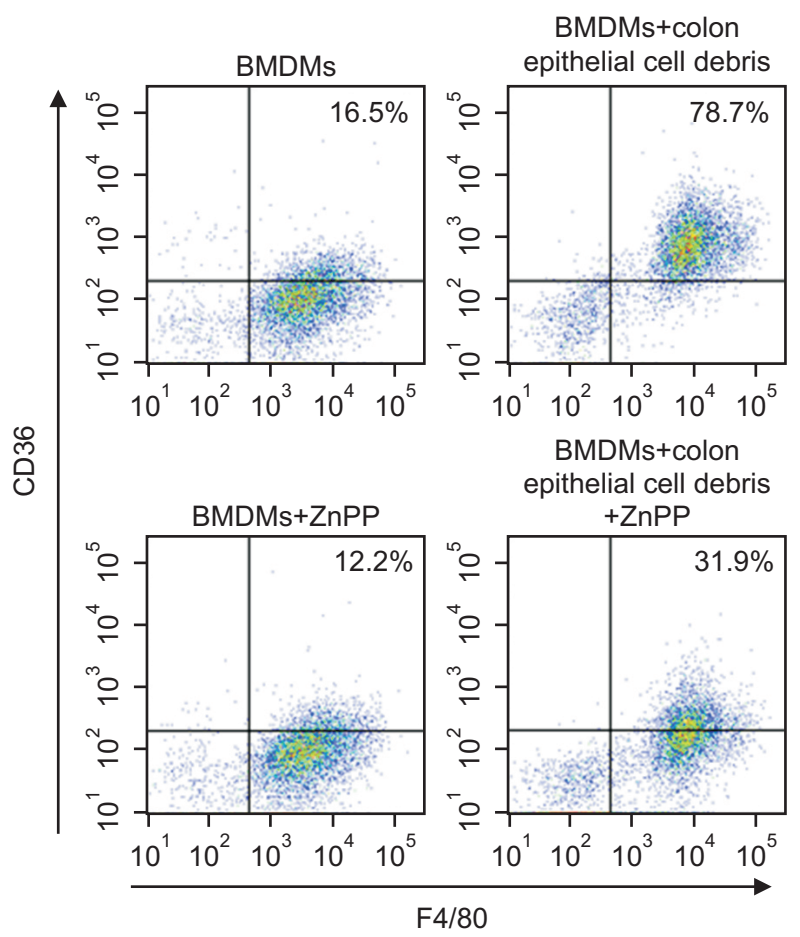

B

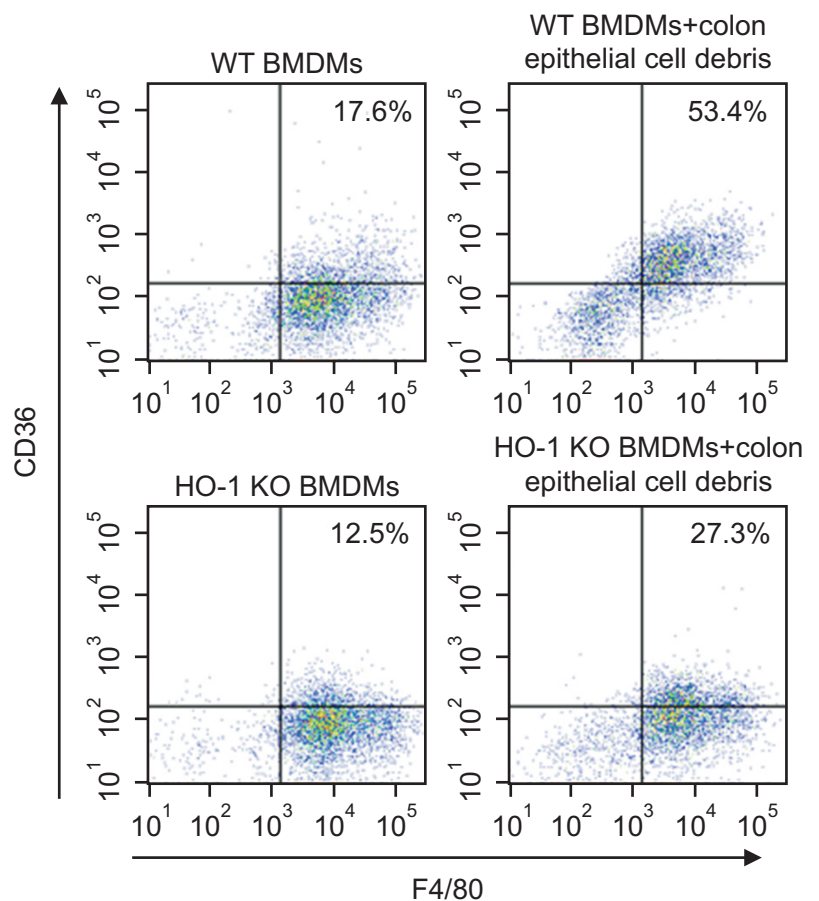

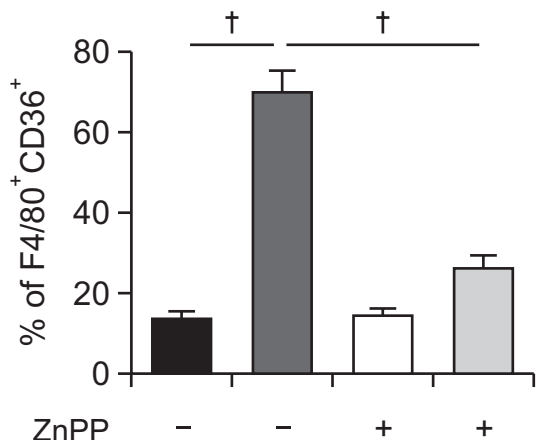

Colon epithelial cell debris

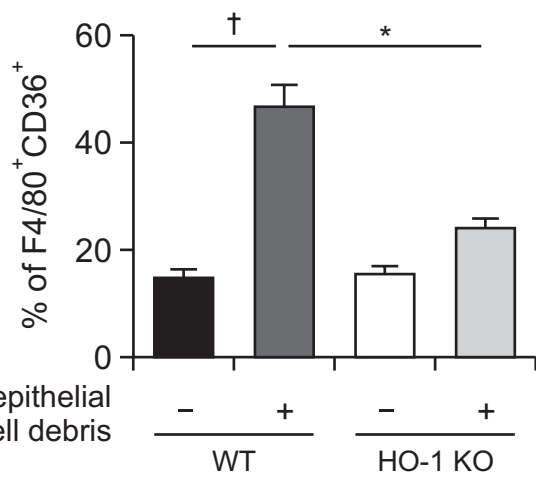

Fig. 7. Role of heme oxygenase-1 (HO-1) in the expression of CD36 in bone marrow-derived macrophages (BMDMs) engulfing apoptotic colonic epithelial cells. CD36 expression in BMDMs mixed with apoptotic epithelial cells in two ways to examine the role of HO-1 in vitro. (A) Murine BMDMs were treated with a pharmacological HO-1 inhibitor, zinc protoporphyrin IX (ZnPP) (10 mM), 1 hour prior to the addition of apoptotic epithelial cells. After incubation for 48 hours, the proportion of BMDMs expressing CD36 was measured by flow cytometry. (B) BMDMs from H0-1 knockout (KO) mice were treated with apoptotic epithelial cell debris and subjected to flow cytometry. Data are expressed as the mean \pm SEM ( $n=3$ ). WT, wild type; SEM, standard error of the mean. ${ }^{*} p<0.01,{ }^{\dagger} p<0.001$.

pression. ${ }^{32,56,57}$ We found that engulfment of inflammatory apoptotic epithelial cells stimulated HO-1 expression in macrophages. HO-1 induction in macrophages phagocy- tosing dead cells is considered to compromise the oxidative stress which often accompanies during acute inflammation. ${ }^{21}$ Notably, pharmacological inhibition of HO-1 
activity delayed the resolution of DSS-induced colitis by hampering the M2 macrophage polarization.

In agreement with our observations, administration of an HO-1 inducing agent, hemin ameliorated intestinal inflammation and remedied intestinal mucosal barrier damage by correcting abnormal intestinal macrophage polarization. In addition, hemin inhibited the M1 polarization while enhancing M2 polarization in BMDMs, and these effects were abrogated by silencing $\mathrm{HO}-1{ }^{58}$ The above findings suggest the therapeutic potential of HO-1 inducers capable of modulating the macrophage functions in the management of IBD. Based on the known contribution of macrophages to intestinal homeostasis, macrophage therapy has been proposed as a means of treating IBD. The transferring purified bone marrow-derived M2 macrophages to mice subjected to DSS-induced colitis was shown to attenuate disease severity. ${ }^{59}$ Under the same principle, effectively regulating macrophage polarization in vivo may become a potential therapeutic strategy for treating UC. ${ }^{60}$

Whilst DSS administration induced HO-1 expression in both entire colonic mucosa and tissue macrophages, their kinetics are apparently different. HO-1 is a stress-responsive enzyme, and its expression in the colonic mucosa is induced relatively rapidly upon acute inflammation caused by DSS as adaptive response. In contrast, HO-1 in colonic macrophages is likely to be induced after they engulf the apoptotic epithelial cells. This may account for the delay in the onset of HO-1 expression in macrophages compared with that in the entire colon. Therefore, the epithelial HO-1 induction does not necessarily represent the macrophagespecific HO-1 increased in a recovery phase, but might rather be a result of inflammatory reaction.

In summary, engulfment of apoptotic colonic epithelial cells significantly induces $\mathrm{HO}-1$ expression in macrophages. The HO-1 induction accelerates the resolution of DSS-induced colitis by inducing polarization of macrophages phagocytosing apoptotic colonic epithelial cells. According to Lee et al., ${ }^{61}$ an elevated accumulation of apoptotic cells resulting from impaired efferocytosis contributes to the excessive and prolonged colonic inflammation. Of interest, restoration of efferocytosis by adoptive transfer of BMDMs to the inflamed colonic tissue promotes resolution of DSS-induced colitis. In this regard, transfer of HO-1 overexpressing macrophages would be a new therapeutic strategy in the management of IBD, which requires clinical validation.

\section{CONFLICTS OF INTEREST}

No potential conflict of interest relevant to this article was reported.

\section{ACKNOWLEDGEMENTS}

This work was supported by the Basic Science Research Program grant (number: 2021R1A2C2014186 to Y.J.S.), the Priority Research Centres Programme (number: 2014R1A6A1030318 to H.T.C.) and the BK21 FOUR Program (number: 5120200513755) from the National Research Foundation, Republic of Korea.

\section{AUTHOR CONTRIBUTIONS}

Conceptualization: S.Y.G., S.H.K., H.N.L. Methodology: S.J.K., J.P., S.H.K., Y.J., H.N.L., W.K., I.A.M. Formal analysis: S.Y.G., S.J.K., J.P., S.H.K., Y.J. Funding acquisition: Y.J.S. Project administration: Y.J.S. Writing - original draft: S.Y.G. Writing - review and editing: Y.J.S. Resources: Y.J., H.T.C., H.K.N. Approval of final manuscript: all authors.

\section{ORCID}

Shin-Young Gwak

Su-Jung Kim

Jeongmin Park

https://orcid.org/0000-0002-8988-7087 https://orcid.org/0000-0002-3636-0644 https://orcid.org/0000-0002-3243-7800

Seung Hyeon Kim https://orcid.org/0000-0002-9359-7517 Yeonsoo Joe https://orcid.org/0000-0001-8407-8902 Ha-Na Lee https://orcid.org/0000-0001-5063-6689 Wonki Kim https://orcid.org/0000-0003-0339-7675 Ishrat Aklima Muna https://orcid.org/0000-0003-4384-1611 Hye-Kyung Na https://orcid.org/0000-0003-0460-2810 Hun Taeg Chung https://orcid.org/0000-0003-4802-7467 Young-Joon Surh https://orcid.org/0000-0001-8310-1795

\section{SUPPLEMENTARY MATERIALS}

Supplementary materials can be accessed at https://doi. org/10.5009/gnl210058.

\section{REFERENCES}

1. Fullerton JN, Gilroy DW. Resolution of inflammation: a new therapeutic frontier. Nat Rev Drug Discov 2016;15:551-567.

2. Sansbury BE, Spite M. Resolution of acute inflammation and the role of resolvins in immunity, thrombosis, and vascular biology. Circ Res 2016;119:113-130. 
3. Ortega-Gómez A, Perretti M, Soehnlein O. Resolution of inflammation: an integrated view. EMBO Mol Med 2013;5:661-674.

4. Schett G, Neurath MF. Resolution of chronic inflammatory disease: universal and tissue-specific concepts. Nat Commun 2018;9:3261.

5. Kaser A, Zeissig S, Blumberg RS. Inflammatory bowel disease. Annu Rev Immunol 2010;28:573-621.

6. Saleh M, Trinchieri G. Innate immune mechanisms of colitis and colitis-associated colorectal cancer. Nat Rev Immunol 2011;11:9-20.

7. Farache J, Zigmond E, Shakhar G, Jung S. Contributions of dendritic cells and macrophages to intestinal homeostasis and immune defense. Immunol Cell Biol 2013;91:232-239.

8. Nunes T, Bernardazzi C, de Souza HS. Cell death and inflammatory bowel diseases: apoptosis, necrosis, and autophagy in the intestinal epithelium. Biomed Res Int 2014;2014:218493.

9. Coskun M. Intestinal epithelium in inflammatory bowel disease. Front Med (Lausanne) 2014;1:24.

10. Murray PJ, Wynn TA. Protective and pathogenic functions of macrophage subsets. Nat Rev Immunol 2011;11:723-737.

11. Eissa N, Hussein H, Kermarrec L, et al. Chromofungin ameliorates the progression of colitis by regulating alternatively activated macrophages. Front Immunol 2017;8:1131.

12. Biswas A, Shouval DS, Griffith A, et al. WASP-mediated regulation of anti-inflammatory macrophages is IL-10 dependent and is critical for intestinal homeostasis. Nat Commun 2018;9:1779.

13. Hayashi S, Hamada T, Zinsou DGA, et al. PI3K p85a subunit-deficient macrophages protect mice from acute colitis due to the enhancement of IL-10 production. Sci Rep 2017;7:6187.

14. Kayama H, Kohyama M, Okuzaki D, et al. Heme ameliorates dextran sodium sulfate-induced colitis through providing intestinal macrophages with noninflammatory profiles. Proc Natl Acad Sci U S A 2018;115:8418-8423.

15. Denning TL, Wang YC, Patel SR, Williams IR, Pulendran B. Lamina propria macrophages and dendritic cells differentially induce regulatory and interleukin 17-producing $\mathrm{T}$ cell responses. Nat Immunol 2007;8:1086-1094.

16. Savill J, Dransfield I, Gregory C, Haslett C. A blast from the past: clearance of apoptotic cells regulates immune responses. Nat Rev Immunol 2002;2:965-975.

17. Kawano M, Nagata S. Efferocytosis and autoimmune disease. Int Immunol 2018;30:551-558.

18. Das KM, Biancone L. Is IBD an autoimmune disorder? Inflamm Bowel Dis 2008;14 Suppl 2:S97-S101.

19. Scott RS, McMahon EJ, Pop SM, et al. Phagocytosis and clearance of apoptotic cells is mediated by MER. Nature 2001;411:207-211.
20. Zhang S, Weinberg S, DeBerge M, et al. Efferocytosis fuels requirements of fatty acid oxidation and the electron transport chain to polarize macrophages for tissue repair. Cell Metab 2019;29:443-456.

21. Cunha LD, Yang M, Carter R, et al. LC3-associated phagocytosis in myeloid cells promotes tumor immune tolerance. Cell 2018;175:429-441.

22. Elliott MR, Koster KM, Murphy PS. Efferocytosis signaling in the regulation of macrophage inflammatory responses. J Immunol 2017;198:1387-1394.

23. Chiang N, Shinohara M, Dalli J, et al. Inhaled carbon monoxide accelerates resolution of inflammation via unique proresolving mediator-heme oxygenase-1 circuits. J Immunol 2013;190:6378-6388.

24. Gozzelino R, Jeney V, Soares MP. Mechanisms of cell protection by heme oxygenase-1. Annu Rev Pharmacol Toxicol 2010;50:323-354.

25. Vijayan V, Wagener FADTG, Immenschuh S. The macrophage heme-heme oxygenase-1 system and its role in inflammation. Biochem Pharmacol 2018;153:159-167.

26. Sheikh SZ, Hegazi RA, Kobayashi T, et al. An anti-inflammatory role for carbon monoxide and heme oxygenase-1 in chronic Th2-mediated murine colitis. J Immunol 2011;186:5506-5513.

27. Tzima S, Victoratos P, Kranidioti K, Alexiou M, Kollias G. Myeloid heme oxygenase-1 regulates innate immunity and autoimmunity by modulating IFN-beta production. J Exp Med 2009;206:1167-1179.

28. Hegazi RA, Rao KN, Mayle A, Sepulveda AR, Otterbein LE, Plevy SE. Carbon monoxide ameliorates chronic murine colitis through a heme oxygenase 1-dependent pathway. J Exp Med 2005;202:1703-1713.

29. Marelli G, Erreni M, Anselmo A, et al. Heme-oxygenase-1 production by intestinal CX3CR1+ macrophages helps to resolve inflammation and prevents carcinogenesis. Cancer Res 2017;77:4472-4485.

30. Onyiah JC, Sheikh SZ, Maharshak N, Otterbein LE, Plevy SE. Heme oxygenase- 1 and carbon monoxide regulate intestinal homeostasis and mucosal immune responses to the enteric microbiota. Gut Microbes 2014;5:220-224.

31. Zhang M, Nakamura K, Kageyama S, et al. Myeloid HO-1 modulates macrophage polarization and protects against ischemia-reperfusion injury. JCI Insight 2018;3:e120596.

32. Gobert AP, Verriere T, Asim M, et al. Heme oxygenase-1 dysregulates macrophage polarization and the immune response to Helicobacter pylori. J Immunol 2014;193:30133022.

33. Naito Y, Takagi T, Higashimura Y. Heme oxygenase-1 and anti-inflammatory M2 macrophages. Arch Biochem Biophys 2014;564:83-88.

34. Erben U, Loddenkemper C, Doerfel K, et al. A guide to his- 
tomorphological evaluation of intestinal inflammation in mouse models. Int J Clin Exp Pathol 2014;7:4557-4576.

35. Weigmann B, Tubbe I, Seidel D, Nicolaev A, Becker C, Neurath MF. Isolation and subsequent analysis of murine lamina propria mononuclear cells from colonic tissue. Nat Protoc 2007;2:2307-2311.

36. Chassaing B, Aitken JD, Malleshappa M, Vijay-Kumar M. Dextran sulfate sodium (DSS)-induced colitis in mice. Curr Protoc Immunol 2014;104:15.25.1-15.25.14.

37. Wirtz S, Popp V, Kindermann M, et al. Chemically induced mouse models of acute and chronic intestinal inflammation. Nat Protoc 2017;12:1295-1309.

38. Sebastián VP, Salazar GA, Coronado-Arrázola I, et al. Heme oxygenase- 1 as a modulator of intestinal inflammation development and progression. Front Immunol 2018;9:1956.

39. Yakoub AM, Schulz R, Seiffert M, Sadek M. Autoantigenharboring apoptotic cells hijack the coinhibitory pathway of T cell activation. Sci Rep 2018;8:10533.

40. Kim W, Kim HU, Lee HN, et al. Taurine chloramine stimulates efferocytosis through upregulation of Nrf2-mediated heme oxygenase- 1 expression in murine macrophages: possible involvement of carbon monoxide. Antioxid Redox Signal 2015;23:163-177.

41. Kim SH, Zhong X, Kim W, et al. Taurine chloramine potentiates phagocytic activity of peritoneal macrophages through up-regulation of dectin-1 mediated by heme oxygenase1-derived carbon monoxide. FASEB J 2018;32:2246-2257.

42. Lee CS, Penberthy KK, Wheeler KM, et al. Boosting apoptotic cell clearance by colonic epithelial cells attenuates inflammation in vivo. Immunity 2016;44:807-820.

43. Parks BW, Black LL, Zimmerman KA, et al. CD36, but not G2A, modulates efferocytosis, inflammation, and fibrosis following bleomycin-induced lung injury. J Lipid Res 2013;54:1114-1123.

44. Oz HS, Zhong J, de Villiers WJ. Pattern recognition scavenger receptors, SR-A and CD36, have an additive role in the development of colitis in mice. Dig Dis Sci 2009;54:25612567.

45. Okayasu I, Hatakeyama S, Yamada M, Ohkusa T, Inagaki Y, Nakaya R. A novel method in the induction of reliable experimental acute and chronic ulcerative colitis in mice. Gastroenterology 1990;98:694-702.

46. Bain CC, Schridde A. Origin, differentiation, and function of intestinal macrophages. Front Immunol 2018;9:2733.

47. Lavin Y, Winter D, Blecher-Gonen R, et al. Tissue-resident macrophage enhancer landscapes are shaped by the local microenvironment. Cell 2014;159:1312-1326.

48. Davies LC, Jenkins SJ, Allen JE, Taylor PR. Tissue-resident macrophages. Nat Immunol 2013;14:986-995.

49. Serbina NV, Pamer EG. Monocyte emigration from bone marrow during bacterial infection requires signals mediated by chemokine receptor CCR2. Nat Immunol 2006;7:311317.

50. Bain CC, Scott CL, Uronen-Hansson H, et al. Resident and pro-inflammatory macrophages in the colon represent alternative context-dependent fates of the same Ly6Chi monocyte precursors. Mucosal Immunol 2013;6:498-510.

51. Rugtveit J, Nilsen EM, Bakka A, Carlsen H, Brandtzaeg P, Scott H. Cytokine profiles differ in newly recruited and resident subsets of mucosal macrophages from inflammatory bowel disease. Gastroenterology 1997;112:1493-1505.

52. Vinuesa E, Hotter G, Jung M, Herrero-Fresneda I, Torras J, Sola A. Macrophage involvement in the kidney repair phase after ischaemia/reperfusion injury. J Pathol 2008;214:104113.

53. Zhu W, Yu J, Nie Y, et al. Disequilibrium of M1 and M2 macrophages correlates with the development of experimental inflammatory bowel diseases. Immunol Invest 2014;43:638652.

54. Horuluoglu BH, Kayraklioglu N, Tross D, Klinman D. PAM3 protects against DSS-induced colitis by altering the M2:M1 ratio. Sci Rep 2020;10:6078.

55. Kim E, Kim Y, Lee J, et al. Leucrose, a natural sucrose isomer, suppresses dextran sulfate sodium (DSS)-induced colitis in mice by regulating macrophage polarization via JAK1/ STAT6 signaling. J Funct Foods 2020;74:104156.

56. Camhi SL, Alam J, Otterbein L, Sylvester SL, Choi AM. Induction of heme oxygenase-1 gene expression by lipopolysaccharide is mediated by AP-1 activation. Am J Respir Cell Mol Biol 1995;13:387-398.

57. Shiloh MU, Manzanillo P, Cox JS. Mycobacterium tuberculosis senses host-derived carbon monoxide during macrophage infection. Cell Host Microbe 2008;3:323-330.

58. Wu Y, Wu B, Zhang Z, et al. Heme protects intestinal mucosal barrier in DSS-induced colitis through regulating macrophage polarization in both $\mathrm{HO}-1$-dependent and $\mathrm{HO}$ 1-independent way. FASEB J 2020;34:8028-8043.

59. Hunter MM, Wang A, Parhar KS, et al. In vitro-derived alternatively activated macrophages reduce colonic inflammation in mice. Gastroenterology 2010;138:1395-1405.

60. Sun T, Kwong CHT, Gao C, et al. Amelioration of ulcerative colitis via inflammatory regulation by macrophage-biomimetic nanomedicine. Theranostics 2020;10:10106-10119.

61. Lee HN, Tian L, Bouladoux N, et al. Dendritic cells expressing immunoreceptor CD300f are critical for controlling chronic gut inflammation. J Clin Invest 2017;127:1905-1917. 